\title{
Ativação do paciente com diagnóstico de doença renal crônica: uma revisão integrativa
}

\author{
Denise Rocha Raimundo-Leone ${ }^{1}$, Arison Cristian de Paula-Silva ${ }^{2}$, Aline Silva-de Aguiar ${ }^{1}$ \\ ${ }^{1}$ Universidade Federal de Juiz de Fora. Programa de Pós Graduação em Saúde Coletiva. Minas Gerais. Brasil \\ ${ }^{2}$ Universidade do Estado do Rio de Janeiro. Residência de Enfermagem em Nefrologia. Rio de Janeiro. Brasil
}

Como citar este artículo:

Raimundo-Leone DR, de Paula-Silva AC, Silva-de Aguiar A. Ativação do paciente com diagnóstico de doença renal crônica: uma revisão integrativa. Enferm Nefrol. 2021 Ene-Mar;24(1):11-23

\section{Resumo}

Objetivo: Estabelecer a medida de ativação de pacientes com doença renal crônica e seus fatores associados.

Metodologia: Revisão integrativa de literatura realizada nas seguintes bases de dados: Pubmed, Lilacs, Scielo, Scopus, Science Direct e Insignia Health. Foram utilizados os descritores: Patient Activation, Nephrology, Kidney e Renal Insufficiency, Chronic e seus correspondentes em português e espanhol e os operadores booleanos AND e OR. Não foi utilizado filtro de data para as buscas. Nove manuscritos compuseram a amostra final desta revisão.

Resultados: A média do escore de ativação variou entre $51 \pm 10$ e $65,02 \pm 16$ pontos, com o predomínio de pessoas com alta ativação (níveis 3 e 4). A ativação do paciente foi associada a múltiplas variáveis, entre elas a idade, condição socioeconômica, escolaridade e carga de sintomas.

Conclusões: Conhecer o nível de ativação dos pacientes e os fatores associados a ele direciona a atuação do profissional de saúde para obtenção e/ou manutenção da alta ativação.

PALAVRAS-CHAVE: ativação; autogestão; doença renal crônica; nefrologia.

\section{Correspondencia:}

Denise Rocha Raimundo Leone

E-mail: de_rocha@ymail.com
Activación del paciente diagnosticado de enfermedad renal crónica: una revisión integrativa

\section{Resumen}

Objetivo: Conocer las medidas de activación empleadas por los pacientes con enfermedad renal crónica y los factores asociados.

Metodología: Revisión integrativa de la literatura realizada en las siguientes bases de datos: Pubmed, Lilacs, Scielo, Scopus, Science Direct e Insignia Health. Se utilizaron los descriptores: Patient Activation, Nephrology, Kidney and Renal Insufficiency, Chronic así como los términos en portugués y español y los operadores booleanos AND y OR. No se utilizó ningún filtro de fecha para las búsquedas. Nueve manuscritos conformaron la muestra final de esta revisión.

Resultados: El puntaje promedio de activación varió entre $51 \pm 10$ y $65,02 \pm 16$ puntos, con predominio de personas con alta activación (niveles 3 y 4). La activación del paciente se asoció con múltiples variables tales como la edad, el nivel socioeconómico, la educación y la carga de los síntomas.

Conclusiones: Conocer el nivel de activación de los pacientes y los factores asociados ayuda al profesional de la salud a la obtención y/o mantenimiento de una alta activación.

PALABRAS CLAVE: activación; nefrología; insuficiencia renal crónica; autogestión. 


\section{Activation of the patient diagnosed with chronic kidney disease: an integrative review}

\begin{abstract}
Objective: To know the activation measures used by patients with chronic kidney disease and the associated factors.

Methodology: Integrative literature review carried out in the following databases: Pubmed, Lilacs, Scielo, Scopus, Science Direct and Insignia Health. The descriptors were used: Patient Activation, Nephrology, Kidney and Renal Insufficiency, Chronic as well as the terms in Portuguese and Spanish and the Boolean operators AND and OR. No date filter was used for searches. Nine manuscripts made up the final sample of this review.
\end{abstract}

Results: The average activation score varied between $51 \pm 10$ and $65.02 \pm 16$ points, with a predominance of people with high activation (levels 3 and 4). Patient activation was associated with multiple variables such as age, socioeconomic status, education and symptom load.

Conclusions: Knowing the level of activation of patients and the associated factors helps the health professional to obtain and/or maintain a high activation.

KEYWORDS: activation; nephrology; renal insufficiency chronic; self-management.

\section{Introdução}

A Doença Renal Crônica (DRC) é considerada um problema de saúde pública global. No Brasil, considerando todos os estágios da doença, estima-se que haja de 3 a 6 milhões de pessoas com DRC. Destes, 122.825 realizam tratamento dialítico. Há um predomínio dos indivíduos que realizam hemodiálise (HD) $(92 \%)$ quando comparada à diálise peritoneal (DP) $(8 \%)^{1,2}$.

A DRC e as doenças associadas corresponderam a $1,82 \%$ e $5,79 \%$, respectivamente, de todas as causas de internações hospitalares no Brasil, sendo estas responsáveis por $2,87 \%$ e $10,10 \%$ de todas as despesas do Sistema Único de Saúde (SUS) nos anos de 2013 a $2015^{3}$.

Para além dos custos financeiros, a DRC ocasiona uma série de alterações bioquímicas, clínicas e metabólicas nos indivíduos. Estas acarretam impactos fisiológicos e emocionais, além de danos nas questões profissionais, sociais, sexuais e psicológicas. Essa situação pode gerar estresse para os pacientes, o que reflete negativamente na qualidade de vida $(\mathrm{QV})^{4,5}$.

Dessa forma, tornam-se necessárias alternativas que visem ao diagnóstico precoce e/ou que retardem a evolução da DRC ${ }^{3}$. Considerando que baixos níveis de ativação são indicadores de progressão para doenças crônicas, como o diabetes mellitus e a depressão, estabelecer estratégias que aumentem a ativação pode trazer benefícios para os indivíduos com DRC, suas famílias e para os sistemas de saúde ${ }^{6-8}$.

Nesse contexto, conhecer o nível de ativação e seus fatores associados pode subsidiar a atuação dos profissionais de saúde na organização dos cuidados aos pacientes com DRC. Pois, assim, podem-se identificar os pacientes com maiores chances de baixa ativação e atuar junto a eles, por meio de intervenções adaptadas ao nível de ativação, para a obtenção de um autogerenciamento eficaz ${ }^{6,9}$.

Salienta-se que a educação para a autogestão deve ser realizada de modo colaborativo e centrado no paciente, sendo este um princípio da autogestão das doenças crônicas ${ }^{10}$ e, ao considerar a classificação da ativação por nível, favorece-se a educação centrada no indivíduo ${ }^{11}$.

A ativação do paciente se refere à capacidade de autogestão da saúde, na qual o indivíduo é inserido no processo de cuidado, que considera os conhecimentos, as habilidades e a motivação do indivíduo para o gerenciamento do seu cuidado de saúde ${ }^{11-12}$. A ativação pode ser mensurada através de uma escala denominada $P a-$ tient Activation Measure (PAM), que possui 13 itens. Trata-se de uma escala comercial que fornece uma visão sobre os indivíduos e suas características, comportamentos e motivações relacionados à saúde ${ }^{11,13}$.

A PAM-13 resulta no escore de ativação e, por meio dele, classifica-se 0 indivíduo em quatro níveis ${ }^{11,13,14}$. 0 nível 1 é muito baixo, os indivíduos são passivos e não têm confiança. Além disso, possuem baixo conhecimento sobre sua saúde. No nível 2, nível baixo, os indivíduos 
possuem algum conhecimento sobre sua saúde, entretanto ainda permanecem muitas lacunas. Os pacientes classificados como nível 2 acreditam que grande parte de sua saúde se encontra fora do seu controle, contudo eles conseguem estabelecer simples objetivos. Já no nível 3, nível médio, que é considerado o nível do agir, os indivíduos estão em processo de desenvolvimento de habilidades de autogestão, além disso, eles se esforçam para manter os melhores comportamentos e se guiam por objetivos. E, no nível 4, nível alto, os indivíduos adotam atividades saudáveis e seguem as recomendações. Para esses pacientes, o foco é a manutenção de um estilo de vida saudável.

Sendo assim, pode-se afirmar que indivíduos com baixa ativação não compreendem a importância do seu papel no gerenciamento da saúde, apresentando lacunas de conhecimentos e limitação da autogestão. Já pessoas com alta ativação tendem a ser mais proativas em relação à própria saúde, além de desenvolverem habilidades de autogerenciamento mesmo em situações de estresse ou mudança ${ }^{13}$.

Há evidências de que maiores escores de ativação do paciente foram relacionados a menores custos com saúde, comportamentos benéficos, maior confiança na gestão de saúde, uso racional dos serviços de saúde e melhoria nos marcadores clínicos. Salienta-se que, ao enfatizar a ativação do paciente, se estabelece um futuro sistema de saúde mais sustentável12,15,16.

Em estudo longitudinal, realizado com 58.277 pessoas com diabetes, pré-diabetes e sem diabetes, constatou-se que pacientes com nível 2 de PAM eram 23\% menos propensos a desenvolver pré-diabetes em relação aos pacientes com nível 1 de PAM. Os pacientes com pré-diabetes classificados como nível 4 de PAM apresentavam metade das chances de internação quando comparados aos indivíduos PAM 1. Além disso, 0 nível PAM foi preditivo de valores adequados de colesterol, pressão arterial e triglicérides em pacientes com diabetes e pré-diabetes ${ }^{7}$.

Nesse sentido, há um consenso de que a ativação e o envolvimento do paciente são importantes para melhorar os cuidados de saúde e qualidade do atendimento ${ }^{17-18}$. Sendo assim, com a finalidade de sintetizar evidências que direcionem a atuação profissional junto ao indivíduo, esta revisão tem como objetivo estabelecer a medida de ativação de pacientes com DRC e seus fatores associados.

\section{Metodologia}

Trata de uma revisão integrativa de literatura, de caráter descritivo, que sintetiza os resultados de pesquisas e direciona a prática baseada em evidências. Percorreram-se seis fases, a saber: elaboração da pergunta norteadora, busca na literatura, seleção dos artigos, leitura e análise crítica dos estudos incluídos na revisão, discussão dos resultados e apresentação da revisão ${ }^{19}$.

\section{Elaboração da questão norteadora}

Com o objetivo de revisar a literatura científica nacional e internacional, a fim de estabelecer a ativação dos pacientes com DRC e fatores associados ao nível de ativação destes, elaboraram-se as seguintes questões norteadoras: qual é o nível de ativação dos pacientes com o diagnóstico de doença renal crônica? Quais são os fatores associados ao nível de ativação nessa população?

\section{Busca na literatura}

Os manuscritos foram coletados em outubro de 2019, nas seguintes bases de dados: Literatura Latino-Americano e do Caribe em Ciências da Saúde (Lilacs), Medical Literature Analysis and Retrievel System Online (Medline) através do Pubmed, Scientific Electronic Library Online (Scielo), Science Direct, Scopus e Insignia Health.

Para a busca on-line, foram utilizados os descritores: ativação do paciente (Patient Activation; Activación del paciente), nefrologia (Nephrology; nefrología), rim (Kidney; riñón) e insuficiência renal crônica (Renal insufficiency, chronic; insuficiencia renal crónica). Ademais, os operadores booleanos AND e OR foram empregados.

As chaves de buscas adotadas no Lilacs, Medline, Scielo, Science Direct, Scopus foram: "ativação do paciente" OR "Patient Activation" OR "Activación del paciente") AND ("Nefrologia" OR "Nephrology" OR "Nefrología"); ("Patient Activation" OR "Activación del paciente") AND ("Rim" OR "Kidney" OR "Riñón"); ("ativação do paciente" OR "Patient Activation" OR "Activación del paciente") AND ("Insuficiência Renal Crônica" OR "Renal Insufficiency, Chronic" OR "Insuficiencia Renal Crónica"). Já no Insignia Health, por se tratar de uma base de dados que reúne estudos com o instrumento PAM, utilizaram-se as palavras-chaves na língua inglesa isoladamente, sendo: "Nephrology"; "kidney"; "Renal insufficiency, chronic". 
Os critérios de inclusão definidos foram: ser um estudo primário publicado nas línguas portuguesa, inglesa e espanhola, com resumos que descrevessem o nível de ativação de pacientes com DRC. Não foi utilizado filtro para data de publicação do manuscrito. Adotaram-se como critérios de exclusão: manuscritos que não respondem às questões norteadoras desta revisão, artigos de revisão e aqueles não disponíveis on-line e na íntegra.

\section{Seleção dos artigos}

Considerando os critérios de inclusão, 156 manuscritos foram encontrados, sendo 120 na Science Direct, 14 na Insignia Health, 11 na Medline e 11 na Scopus. Nas demais bases de dados, não foram encontrados manuscritos. Destes, nove estavam presentes nas duas ou mais bases de dados. Sendo assim, 147 artigos constituíram a amostra inicial da revisão.

Num primeiro momento, foi realizada a leitura de título e resumo dos mesmos, para saber se eles respondiam às questões norteadoras. Nos casos de incerteza da elegibilidade do artigo, optou-se pela inclusão para leitura na íntegra. Após essa etapa, 134 artigos foram excluídos, restando 13, que foram considerados para a leitura na íntegra; destes, quatro foram excluídos por não abordarem o nível de ativação de pacientes com DRC e/ou em tratamento dialítico. Sendo assim, a amostra final desta conteve nove manuscritos. A síntese do processo de seleção dos manuscritos encontra-se na Figura 1.

A síntese da análise dos dados foi realizada no Tabela $\mathbf{1}$, onde foram inclusos os seguintes tópicos: autoria, ano e periódico de publicação, tipo de estudo, amostra, medida de ativação e/ou nível de ativação. Todas as publicações foram realizadas na língua inglesa. A amostra contou com sete estudos transversais, um estudo de coorte e um estudo clínico randomizado. No que tange ao local de realização dos estudos, três foram realizados nos Estados Unidos da América ${ }^{21-23}$, três na Austrália ${ }^{17,24-25}$, um na Bélgica ${ }^{26}$, um na Holanda9 e um na Inglaterra ${ }^{27}$. Não foram encontrados manuscritos brasileiros.

Mesmo não tendo utilizado filtro de data para as publicações como critério de inclusão, curiosamente, todos os manuscritos foram publicados nos últimos cinco anos, sendo dois em $2015^{9,22}$ e $2017^{17,25}$, quatro no ano de $2018^{21,22,24,26}$ e um no ano de $2019^{27}$.

Acredita-se que o recente interesse na investigação de ativação de pessoas com DRC tenha sido estimulado pelo fato de a PAM ter sido endossada como uma medida de cuidado centrada na pessoa pelo National Quality Forum

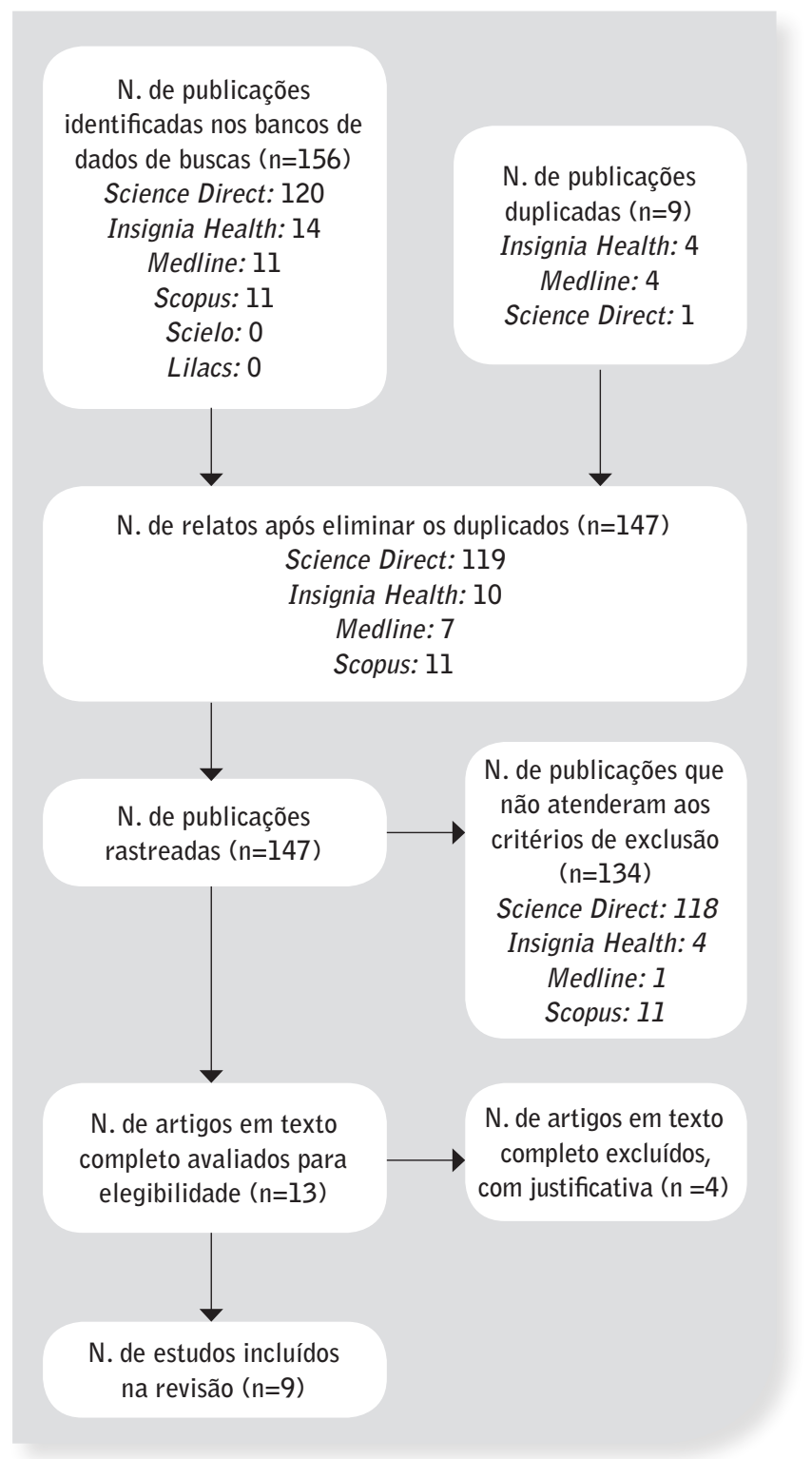

Figura 1. Síntese do processo de seleção dos manuscritos adaptado do Preferred Reporting Items for Systematic Review and MetaAnalyses (PRISMA)20, Juiz de Fora-Minas Gerais, 2020.

Fonte: os autores.

(NQF) no ano de $2016^{28}$, o que demonstra que se trata de um conhecimento novo, que está em evolução.

\section{Medida de ativação dos pacientes com DRC}

A média do escore basal de ativação variou entre $51,4 \pm 10$ e $65,02 \pm 16$ pontos $9,17,21,22,24,26$. Houve predomínio de pacientes com alta ativação (níveis 3 e 4) em detrimento dos pacientes classificados com baixa ativação (nível 1 e 2), nos estudos de Nelson et al. (2018) (84\% 
Tabela 1. Síntese das características dos estudos incluídos na revisão integrativa de literatura, Juiz de Fora-Minas Gerais, 2020.

\begin{tabular}{|c|c|c|c|}
\hline $\begin{array}{l}\text { Autoria, Ano e } \\
\text { periódico }\end{array}$ & Tipo de estudo & Amostra & $\begin{array}{l}\text { Medida de ativação do paciente } \\
\text { (PAM-13) / Nível de ativação }\end{array}$ \\
\hline $\begin{array}{l}\text { Bos-Touwen et al., } \\
2015^{9} \text {. } \\
\text { Plos One }\end{array}$ & $\begin{array}{l}\text { Estudo transversal } \\
\text { descritivo }\end{array}$ & $\begin{array}{c}\text { Estudo com } 1.154 \text { pessoas diagnos- } \\
\text { ticadas com várias doenças crônicas. } \\
\text { Destas, } 219 \text { tinham } D R C(T F G<60 \\
\mathrm{mL} / \mathrm{min} / 1,73 \mathrm{~m}^{2} \text {.) }\end{array}$ & $\begin{array}{l}\text { Resultado referente aos } 219 \text { pacientes com } \\
\text { DRC: PAM-13:51,4 } \pm 10 \text {. }\end{array}$ \\
\hline $\begin{array}{l}\text { Zimbudzi et al., } \\
2017^{* 17} \text {. } \\
\text { BMJ Open }\end{array}$ & Estudo transversal & $\begin{array}{c}305 \text { pessoas com diagnóstico de } \\
\text { diabetes mellitus I e II e TFG }<60 \mathrm{~mL} / \\
\mathrm{min} / 1,73 \mathrm{~m}^{2} \text {. Destes, } 59 \text { estavam em } \\
\text { diálise }\end{array}$ & $\begin{array}{l}\text { PAM-13:57.6 } \pm 15 \\
\text { Nível } 1=22 \% \text {; nível } 2=23,6 \% \text {, } \\
\text { nível 3=36,4\%; nível } 4=18 \%\end{array}$ \\
\hline $\begin{array}{l}\text { Vélez-Bermúdez et al., } \\
2018^{21} \text {. } \\
\text { Annals of Behavioral } \\
\text { Medicine }\end{array}$ & Estudo transversal & $\begin{array}{l}64 \text { pessoas com } D R C \text {, sendo a } \\
T F G<25 \mathrm{~mL} / \mathrm{min} / 1,73 \mathrm{~m}^{2} \text {, que não } \\
\text { realizassem terapia renal substitutiva }\end{array}$ & PAM-13: média $65,02 \pm 16$ pontos. \\
\hline $\begin{array}{l}\text { Nelson et al., } 2018^{22} \text {. } \\
\text { Clinical Journal of the } \\
\text { American Society of } \\
\text { Nephrology }\end{array}$ & $\begin{array}{l}\text { Ensaio clínico } \\
\text { randomizado }\end{array}$ & $\begin{array}{l}125 \text { pessoas, sendo } 63 \text { no grupo de } \\
\text { intervenção com TFG média de } 105 \\
+31 \mathrm{~mL} / \mathrm{min} / 1,73 \mathrm{~m}^{2} \text { e } 62 \text { no grupo de } \\
\text { cuidados usuais, que tinham TFG média } \\
\text { de } 101+2925 \mathrm{~mL} / \mathrm{min} / 1,73 \mathrm{~m}^{2}\end{array}$ & $\begin{array}{c}\text { PAM-13 no grupo de cuidados usuais: Medida } \\
\text { pré: } 65,1 \pm 14,6 ; \text { Pacientes com alta ativação: } \\
84 \% \text { Medida após } 12 \text { meses: } 62,9 \pm 17,1 \\
\text { PAM-13 no grupo de cuidados domiciliares: } \\
\text { Medida pré: } 60,7 \pm 20,5 ; \text { Pacientes com alta } \\
\text { ativação: } 68 \% \text {. Medida após } 12 \text { meses de } \\
\text { intervenção: } 70,3 \pm 21,6\end{array}$ \\
\hline $\begin{array}{l}\text { Johnson et al., } 2015^{23} \text {. } \\
\text { Journal Of Renal Care }\end{array}$ & Estudo transversal & $\begin{array}{l}85 \text { pessoas diagnosticadas com DRC } \\
\text { (todos os estágios, inclusive com } \\
\text { pacientes em diálise), hipertensão e } \\
\text { diabetes mellitus }\end{array}$ & $\begin{array}{c}\text { Nível } 1=10 \% \text {, nível } 2=28 \% \text {, nível 3=28\%, } \\
\text { nível } 4=34 \% \text {. }\end{array}$ \\
\hline $\begin{array}{l}\text { L0 et al., } 2018^{24} \text {. } \\
\text { Journal of Diabetes } \\
\text { and Its Complications }\end{array}$ & $\begin{array}{l}\text { Estudo transversal } \\
\text { multicêntrico }\end{array}$ & $\begin{array}{c}199 \text { pessoas com diabetes mellitus e } \\
\text { DRC, sendo a TFG entre } 20 \text { e } 60 \mathrm{~mL} / \\
\mathrm{min} / 1,73 \mathrm{~m}^{2}\end{array}$ & $\begin{array}{c}\text { PAM-13: } 59,9 \pm 15,2 \text {. Sendo: nível } 1=19,6 \% \text {, } \\
\text { nível 2=22,6\%, nível } 3=29,2 \% \\
\text { e nível } 4=28,6 \%\end{array}$ \\
\hline $\begin{array}{l}\text { Zimbudzi et al., } \\
2017^{* 25} \text {. } \\
\text { Health Expectations }\end{array}$ & Estudo transversal & $\begin{array}{c}\text { Estudo com a mesma população do } \\
\text { anterior (10), sendo } 305 \text { diabéticos e } \\
\text { doentes renais crônicos (TFG<60 mL/ } \\
\min / 1,73 \mathrm{~m}^{2} \text {.) }\end{array}$ & PAM-13:57,6 \pm 15 \\
\hline $\begin{array}{l}\text { Van Bulck et al., } \\
2018^{26} \text {. } \\
\text { Bmc Nephrology }\end{array}$ & Estudo transversal & $\begin{array}{l}192 \text { pessoas com DRC e em } \\
\text { tratamento hemodialítico }\end{array}$ & $\begin{array}{l}\text { PAM-13:51 } \pm 10 \text {. Sendo: nível } 1=44 \% \text {, } \\
\text { nível } 2=29 \% \text {, nível 3=22\% e nível } 4=5 \%\end{array}$ \\
\hline $\begin{array}{l}\text { The Renal Association, } \\
2019^{27} \text {. }\end{array}$ & Coorte & $\begin{array}{l}\text { Estudo com } 3.325 \text { pessoas com DRC } \\
\text { em tratamento por HD, DP, transplante } \\
\text { renal ou tratamento conservador. }\end{array}$ & $\begin{array}{c}\text { Nível } 1=25 \% \text {, nível } 2=18 \% \text {, nível 3=33\%, } \\
\text { nível 4=17\%. Missing:7\% }\end{array}$ \\
\hline
\end{tabular}

TFG: taxa de filtração glomerular; DRC: doença renal crônica; HD: hemodiálise; DP: diálise peritoneal.

*0s estudos possuíam objetivos e variáveis diferentes, contudo utilizaram a mesma população, por esse motivo, apresentam o mesmo valor de PAM-13.

Fonte: 0s autores.

$x 16 \%$, considerando valor basal no grupo de cuidados habituais e $68 \%$ x $32 \%$ no grupo de intervenção, no início do estudo) ${ }^{22}$, LO et al. (2018) $(57,8 \% \times 42,2 \%)^{24}$, Zimbudzi et al. (2017) $(54,4 \% \times 45,6 \%) 17$, Johnson et al. (2015) $(62 \% \times 38 \%)^{23}$ e The Renal Association (2019) $(50 \% \times 43 \%)^{27}$.
Em contrapartida, no estudo de Van Bulck et al. (2018), $73 \%$ dos pacientes foram classificados como baixa ativação ${ }^{26}$. Vale ressaltar que esse foi o único manuscrito que avaliou exclusivamente a ativação de pacientes em HD. Os pacientes que realizam o tratamento hemodialítico também possuíram níveis de ativação inferiores 
(52\% tinham baixos níveis de ativação) quando comparados a pessoas que realizam diálise peritoneal (32\%), aos transplantados renais $(25 \%)$ e àqueles em tratamento conservador da DRC $(40 \%)^{27}$.

No estudo de Velez Bermudez (2018), foi avaliada a média da ativação conforme modalidade de tratamento preferido pelos participantes que se aproximavam da necessidade da Terapia Renal Substitutiva (TRS). Os pacientes que preferiam a DP eram mais ativados (PAM $=75,2)$ quando comparados àqueles que preferiam a HD realizada no centro de diálise (PAM $=66,9)$, HD domiciliar (PAM $=65,1)$ e tratamento conservador (PAM $=55,8)$. Os pacientes que não tinham certeza sobre a preferência de TRS obtiveram o valor de PAM de 61, $5^{21}$. Esse achado corrobora a ideia de que o tipo de TRS realizada influencia o nível de ativação dos pacientes renais crônicos ${ }^{27}$.

Possíveis justificativas para essas diferenças podem estar relacionadas com a natureza do autocuidado que cada modalidade de tratamento para a DRC precisa, além de diferenças quanto a idade, presença e quantidade de comorbidade e tempo de tratamento ${ }^{27}$. 0 fato de que os pacientes em DP necessitam ter habilidades para 0 autocuidado, que os possibilitem realizar o tratamento em domicílio, torna-os mais ativados ${ }^{21}$, o que ratifica a informação de que os níveis de ativação podem variar, consideravelmente, de acordo com a gravidade da doença e seu tratamento ${ }^{29}$.

Contudo, o nível de ativação não aumentou conforme a progressão da DRC e, consequentemente, a diminuição da TFG em estudo americano com 85 pessoas que se encontravam em diferentes estágios da DRC ${ }^{23}$.

Ao analisar a frequência relativa de pacientes que foram classificados como nível 4 de ativação, considerando todos os manuscritos que apresentaram essa variável, apenas $20,52 \%$ dos pacientes tinham nível 4 de ativação, o que permite apreender que há espaço para atuação profissional no desenvolvimento de atividades junto aos pacientes com DRC, que vislumbre a obtenção de alta ativação?.

\section{Fatores associados à ativação de pacientes com doença renal crônica}

A ativação do paciente com DRC foi associada a múltiplas variáveis conforme descrito a seguir:

\section{Idade}

Altas pontuações de ativação foram associadas a uma menor idade em pessoas em tratamento hemodialítico (B:-0,284, $p<0,001)^{26}$. Considerando todos os estágios da DRC, em coorte inglesa com 3.325 pessoas, maiores pontuações no escore de ativação foram mensuradas em indivíduos na faixa etária de 25 a 44 anos e estas diminuíam conforme o aumento da idade $^{27}$.

Corroborando esses achados, em estudo que teve como objetivo identificar os determinantes da ativação e das variáveis associadas à baixa ativação em pessoas com doenças crônicas, entre elas a DRC, na regressão múltipla, verificou-se que altas pontuações na ativação se associaram a menor idade (B:-0,05; IC:-0,11 a 0,01)9. Ademais, em pesquisa com 305 pacientes com DRC e diabetes, a idade avançada foi associada à baixa ativação (níveis 1 e 2) (B:-0,18; Intervalo de confiança (IC): $-0,35$ a 0,01$)^{17}$.

A idade também apresentou relação inversa com o nível de ativação em estudo que envolveu pacientes com hipertensão arterial, diabetes e doença cardíaca isquêmica $(p<0,001)^{30}$ e em estudo com pacientes com insuficiência cardíaca descompensada aguda $(p=0,001)^{31}$. Essa relação pode ser explicada devido à prevalência de sintomas depressivos relacionados às dificuldades funcionais que prejudicam o autogerenciamento de pessoas idosas ${ }^{32}$.

\section{Escolaridade}

Maiores pontuações no escore de ativação foram associadas a moderada escolaridade quando comparada a baixa escolaridade (B:-0,40; IC:-0,17 a 0,90) no estudo de Bos-Touwen e colaboradores, que também demonstrou que a baixa escolaridade está relacionada à má ativação do paciente (nível 1 de ativação) (B:1,41; IC: 1,03 a 1,92$)^{9}$.

Em pessoas que realizavam o tratamento hemodialítico, considerando a análise linear univariável, pacientes com nível superior $(p=0,004)$ e superior não universitário $(p=0,005)$ apresentaram escores mais elevados de ativação quando comparados às pessoas com ensino primário. Contudo, na análise linear múltipla, essa relação não se manteve significativa ${ }^{26}$.

0 nível de escolaridade também foi relacionado à ativação em outros estudos com pessoas com doenças crônicas. Em pesquisa com indivíduos com insuficiência cardíaca, as pessoas menos ativadas eram aquelas com 
menor escolaridade $(p=0,003)$ e, em pacientes com 0 diagnóstico de fibrilação atrial, os maiores níveis de ativação também estavam presentes em pessoas com uma maior escolaridade $(p=0,004)^{33}$.

Portanto, acredita-se que pessoas com maior escolaridade compreendam melhor as prescrições dos profissionais de saúde e, por este motivo, tenham mais facilidade para o autogerenciamento da saúde, o que pode justificar o relacionamento entre essas duas variáveis ${ }^{34}$.

\section{Condição Socioeconômica}

Houve estudos que estabeleceram a relação entre condição econômica e níveis de ativação. Destaca-se que possuir alguma dificuldade financeira aumenta o risco de a pessoa ser classificada como nível 1 de ativação entre os pacientes com doenças crônicas, entre elas a

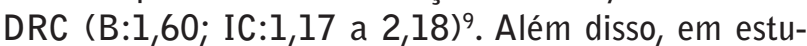
do inglês com pacientes em todos os estágios da DRC, das pessoas com melhores condições socioeconômicas, $63,2 \%(n=233)$ apresentaram alta ativação (níveis 3 ou 4) comparados aos $39,6 \% \quad(n=414)$ dos pacientes com piores condições socioeconômicas $(p<0,001)^{27}$.

Todavia, em estudo que envolveu 305 pessoas com DRC e diabetes comórbido, não se estabeleceu associação significativa entre o nível de ativação e o status socioeconômico ${ }^{17}$. Vale ressaltar que cada estudo utilizou um instrumento para avaliar a condição socioeconômica, o que pode justificar as inconsistências entre nível de ativação e condição socioeconômica.

Contudo, foi observada a significância dessa relação em um estudo chinês, com 509 pacientes com hipertensão e/ou diabetes. Nele, houve diferença significativa no nível de ativação do paciente conforme a renda familiar $(p=0,001)$, o que ratifica 0 achado da associação entre o nível de ativação e a condição crônica ${ }^{35}$.

Entre as variáveis socioeconômicas, altas pontuações no escore de ativação foram associadas a residir com alguém ( $B: 0,141 ; p=0,025)$ entre os pacientes que realizam o tratamento hemodialítico ${ }^{26}$. Corroborando com esse achado, viver sozinho aumenta a chance de a pessoa possuir má ativação (grupo 1 de ativação) entre os pacientes com doenças crônicas, nas quais se inclui a DRC (Odds Ratio (0R):1,50; IC:1,10 a 2,06) ${ }^{9}$. A condição de moradia, se em casa/apartamento ou em outro local, foi apontada como fator que interfere no escore de ativação $(p=0,034)$ de pacientes diabéticos ${ }^{36} .0$ que reitera a associação entre a condição de vida e a ativação.

\section{Realização de atividades de lazer}

A realização de atividades de lazer foi associada a maior escore de ativação nos pacientes que se submetem ao tratamento hemodialítico $(B: 0,206 ; p=0,002)^{26}$. Essa associação pode ser justificada pelo fato de que pessoas com níveis de ativação mais elevados têm menores dificuldades na realização das atividades da vida diária ( $B: 1,14 ; p=0,000)$ e atividades instrumentais da vida diária $(B: 1,14 ; p=0,000)^{37}$, o que pode favorecer a realização de atividades de lazer ${ }^{26}$. Outra possível explicação para essa relação é que pessoas com maiores níveis de ativação são mais propensas a ter um estado de saúde autorreferida melhor $9,26,38$, o que pode contribuir para a realização de atividades de lazer.

\section{Índice de massa corporal}

0 índice de massa corporal (IMC) apresentou uma associação negativa com a ativação no estudo de Bos-Touwen e colaboradores (2015) (B:-0,24; IC:-0,38 a -0,10), o que evidencia que pessoas com um maior IMC são mais propensas a apresentar menor escore de ativação. Reforçando esse achado, um maior IMC foi associado a maior risco de baixa ativação (nível 1), quando comparado aos demais níveis (0R:1,05; IC:1,01 a 1,08)9.

Contudo, no estudo de Zimbudzi e colaboradores (2017), não houve associação significativa entre IMC e nível de ativação ${ }^{17}$. Essa divergência pode estar relacionada à população de estudos, pois a amostragem de Bos-Touwen e colaboradores (2015) foi composta por pacientes com Doença Pulmonar Obstrutiva Crônica (DPOC), Insuficiência Cardíaca Congestiva (ICC), Diabetes Mellitus (DM) tipo II e DRC ${ }^{9}$, já em Zimbudzi e colaboradores (2017), foram participantes pessoas com DRC e diabetes comórbido ${ }^{17}$.

Um IMC maior também foi encontrado em pacientes com nível 1 de ativação em comparação ao nível 4, em estudo com 123 pacientes com fibrilação atrial $(p=0,028)^{33}$. Além disso, em estudo longitudinal com 32.060 adultos atendidos em clínicas de atenção primária, pacientes com níveis de ativação mais altos tinham menor probabilidade de ser obesos quando comparados aos menos ativados ${ }^{15}$.

\section{Realização de atividades de autocuidado}

0 estudo de Zimbudzi e colaboradores (2017) examinou a relação entre a medida de ativação do paciente com a realização das atividades de autocuidado em pessoas com DRC e diabetes. Nele, evidenciou-se que, à medida que diminui o nível de ativação, menor é a pontuação média no escore do composto de autocuidado ${ }^{25}$, que 
é mensurado pelo instrumento Summary of Diabetes Self-Care Activities (SDSCA), que avalia o autogerenciamento do diabetes39 (sendo o nível 4 de ativação como referência, tem-se: nível 1: B:-5,6; IC:-9,5 a -1,8; $\mathrm{p}=<0,01$; nível 2: B:-5,3; IC:-9,1 a -1,8; $\mathrm{p}<0,01$; nível 3: B:-4,1; IC:-7,6 a $-0,6 ; p<0,05)^{25}$.

Além disso, com a diminuição do nível de ativação, a pontuação nas variáveis realização da dieta (nível 4 de ativação como referência, tem-se: nível 1: B:-1,8; IC:3,1 a -0,5; $p=<0,01$; nível 2: B:-1,3; IC:-2,6 a -1,01; $p<0,05)$ e avaliação da glicemia capilar do SDSCA também diminuíram significativamente (nível 4 de ativação como referência, tem-se: nível 1: B:-1,9; IC:-3,5 a -0,4; $p=<0,05$; nível 2: B:-2,1; IC:-3,7 a -0,6; $p<0,01$; nível 3: $B:-1,5 ;$ IC: $-2,9$ a $-0,1 ; p<0,05)$. Diferentemente das variáveis dieta específica, realização de exercícios físicos e cuidados com os pés, que não apresentaram associação significativa com os níveis de ativação ${ }^{25}$.

Corroborando com esses achados, pacientes com má ativação (nível 1) são mais propensos a apresentar menor pontuação no composto autocuidado do instrumento (SDSCA) (B=0,18; IC:0,02 a 0,35; $p<0,05)^{17}$.

Todavia, no estudo de Lo Clement e colaboradores (2018), que também envolveu pessoas com DRC e diabetes, um menor nível de ativação do paciente não se associou significativamente com baixo controle glicêmico e baixo controle da pressão arterial ${ }^{24}$.

Considerando outros grupos amostrais, em estudo americano, pessoas com maior escore de ativação (PAM $=70,5)$ examinavam os pés semanalmente, já aqueles com menores escores de ativação (escore $P A M=65)$ faziam essa verificação mais espaçadamente $(p=0,009)$. Ademais, aqueles pacientes que possuíam maior escore de ativação (PAM $=71,1$ ) fizeram avaliação oftalmológica nos últimos dois anos, conforme recomendado, e aqueles com menor escore $(P A M=66,3)$ ultrapassaram esse tempo ou nunca se submeteram a esse procedimento $(p=0,009) .0$ mesmo aconteceu com a realização de exercício físico, pessoas com prática regular de três vezes na semana ou mais obtiveram média PAM de 71 e, naquelas com frequência menor que três vezes, a média PAM foi de 66,9 $(p=0,021)^{36}$.

Além disso, os indivíduos com maior escore de ativação apresentaram significativamente nenhuma ou pouca dificuldade nos comportamentos de autocuidado com o DM, que eram: administração de medicamentos e/ou in- sulina, escolha de alimentos adequados para a refeição, realização de exercícios regulares, monitorização do nível de glicose sérica, comparecimento às consultas médicas, além de realização de monitoramento de todas as demandas de autocuidado ${ }^{36}$. Isso demonstra a estreita relação entre ativação do paciente e realização das atividades de autocuidado.

\section{Presença de Sintomas}

Há evidências de que níveis mais altos de ativação apresentaram associação com a diminuição da carga de sintomas, considerando cada sintoma individualmente ou a carga geral dos sintomas nos pacientes com DRC, em coorte inglesa. Nesta, $37 \%$ das pessoas que apresentavam baixa ativação (níveis 1 e 2 de ativação) e $20 \%$ dos pacientes altamente ativados (níveis 3 e 4 de ativação) relataram ter apresentado pelo menos um sintoma moderado $(p<0,001)$ no instrumento Palliative care Outcome Scale symptom list for end-stage renal disease (POS-S renal), que considera os seguintes sintomas: vômito, diarreia, náuseas, constipação intestinal, mudanças na pele, inapetência, prurido, boca seca e/ou ferida, síndrome das pernas inquietas, sintomas depressivos, falta de ar, sonolência, sintomas de ansiedade, dor, dificuldade de dormir, mobilidade deficiente e fraqueza ou perda de energia ${ }^{27,40}$.

Corroborando esse achado, maior carga média de sintomas foi relatada por pacientes com fibrilação arterial e classificados como nível 1 quando comparados aos de nível 4 de ativação $(p=0,02)^{33}$. Vale salientar que a associação entre maiores níveis de ativação com a menor presença de sintomas pode ser uma explicação para que pacientes em HD sejam menos ativados, pois há evidências de que estes apresentam uma maior carga de sintomas da doença, quando comparados aos pacientes que realizam outra TRS ${ }^{27}$.

Entretanto, em estudo de Zimbudzi e colaboradores (2017), quando se avaliou o relacionamento entre o componente da qualidade de vida relacionada à saúde (QVRS), lista de sintomas e sua relação com os níveis de ativação, na análise multivariável, esta não foi significativa ${ }^{17}$. Uma possível explicação para essa inconsistência podem ser as diferenças dos instrumentos utilizados, pois um foi específico para sintomas e o outro, um componente da escala que avalia a QVRS.

\section{Depressão}

Há evidências de que os pacientes com maiores escores de ativação foram menos propensos a apresentar depressão (B:-0,14; IC:-0,34 a 0,06). Em contrapartida, 
pacientes com ativação deficiente (nível 1 de ativação) possuem 1,05 chance a mais de apresentar depressão quando comparados aos pacientes com os demais níveis de ativação (0R:1,05; IC:1,01 a 1,10)9.

Contudo, ao analisar a presença de sintomas depressivos, não houve associação significativa entre esta variável e a ativação nos estudos de Van Bulck e colaboradores (2018) e Johnson e colaboradores (2016), diferentemente do encontrado em coorte inglesa, na qual pessoas com menores níveis de ativação estavam mais propensas a apresentar sintomas depres$\operatorname{sivos}^{23,26-27}$. Cabe ressaltar que essas inconsistências podem estar relacionadas às divergências quanto à composição da amostra dos estudos e aos diversos instrumentos de avaliação utilizados.

Salienta-se que, considerando outras populações de pacientes em revisão de escopo que identificou os fatores psicossociais ou psicológicos associados à ativação do paciente, a depressão e os sintomas depressivos apresentaram associação negativa e significativa com os escores de ativação ${ }^{41}$.

\section{Percepção da doença}

Uma melhor saúde autorreferida se associou a maiores escores de ativação em pacientes em tratamento hemodialítico $(B: 0,278 ; p<0,001)^{26}$. Confirmando esse achado, pessoas com maior escore de ativação apresentam menor percepção da doença (B:-0,16; IC:-0,22 a $-0,10$ ) em estudo que envolveu pacientes com doenças crônicas, entre elas a DRC. Nele, os pacientes com nível 1 de ativação possuíram mais chances de perceber a doença (0R:1,03; IC:1,01 a 1,04)9.

Corroborando esses achados, há evidências de que pessoas com DPOC e com ativação deficiente (nível 1 de ativação) apresentam maiores chances de ter uma percepção ruim da doença, o que torna essa uma variável de interesse ao realizar intervenções que visem a altos níveis de ativação ${ }^{34,42}$.

\section{Saúde Física}

Maiores escores de ativação do paciente foram associados positiva e significativamente a melhor saúde física no estudo de Bos-Touwen e colaboradores (2015) (B:0,03; IC:0,00 a 0,006). Contudo, nessa mesma pesquisa, o estado de saúde física não apresentou relação negativa com a má ativação9.

A relação entre a saúde física e o nível de ativação do paciente também foi avaliada em outros estudos, entre- tanto a avaliação da saúde física não se deu por instrumento próprio e sim como componente de escalas que mensuram a QVRS. Nestes, o componente de saúde física ou a função física não apresentaram associação significativa com os níveis de ativação do paciente ${ }^{17,23}$.

\section{Suporte Social}

0 suporte social apresentou associação positiva com a ativação (B:0,15; IC:0,08 a 0,21), no estudo de BosTouwen e colaboradores (2015), o que significa que pessoas com um maior apoio social percebido são mais propícias a possuir escores de ativação mais elevados?. Todavia essa associação não foi significativa nos estudos de Van Bulck e colaboradores (2018) e Johnson e colaboradores (2015), que também envolveram pacientes com DRC, o que demonstra inconsistência nessa relação ${ }^{23,26}$.

Ao explorar outros estudos que abarcassem esse relacionamento, verificou-se que, em revisão sistemática de escopo, o suporte social foi um dos fatores psicossociais associados positiva e significativamente à ativação do paciente, o que demonstrou que o apoio social deve ser avaliado ao realizar intervenções que vislumbrem o aumento dos níveis de ativação do paciente ${ }^{41}$.

\section{Suporte clínico}

No estudo de Van Bulck e colaboradores (2018), pacientes que realizavam tratamento em determinado serviço apresentaram níveis de ativação significativamente menores. Isso demonstrou uma relação negativa entre o estabelecimento de saúde e os escores de ativação (B:-0,45; p:0,019). Uma provável justificativa apontada foram as possíveis diferenças na educação em saúde pré-diálise, pois há evidências de que esta pode ser melhorada por meio dos conhecimentos e das habilidades referentes ao autogerenciamento da doença $a^{26,43-44}$.

Em coorte inglesa com pacientes em todos os estágios da DRC, inclusive em TRS, houve diferenças entre os escores de ativação conforme as unidades de saúde. Contudo os autores sugerem cautela ao avaliarem esses dados, pois essas diferenças podem estar relacionadas a fatores de confusão, como, por exemplo, o tipo de TRS empregado, uma vez que este influencia os níveis de ativação. Além disso, não há garantias de que a amostragem de cada unidade de saúde seja representativa da população atendida nela ${ }^{27}$.

\section{Qualidade de vida relacionada à saúde}

Pacientes com maiores níveis de ativação apresentaram melhor QVRS em todos os cinco domínios do EuroQol five dimension five level index value (EQ5D-5L 
QoL domains ${ }^{27}$, que são mobilidade, autocuidado, atividades usuais, dor e/ou desconforto, ansiedade e/ou depressão $0^{45-46}$, em uma coorte realizada com 3.325 pacientes renais crônicos $(p<0,001)^{27}$.

Em contrapartida, num estudo realizado com 305 pacientes com diabetes e DRC, considerando as cinco subescalas que integram o instrumento Kidney $\mathrm{Di}$ sease Quality of life 36 Items (KDQOL-36), que são: funcionamento físico, funcionamento mental, carga da doença renal, lista de sintomas e problemas e efeitos da $\mathrm{DRC}^{47}$, ter pior saúde autorreferida na subescala de carga da DRC associou-se a menor ativação do paciente (0R:0,11; IC:0,05 a 0,17) ${ }^{17}$. Isso sugere que pacientes mais ativados possuem melhor QVRS.

Corroborando essa afirmação, em estudo no Reino Unido que envolveu pacientes idosos com condições crônicas e multimorbidade, escores de ativação foram significativamente associados a uma melhor QVRS (B:9,56; IC:6,71 a 12,40$)^{48}$. Isso torna a ativação do paciente uma métrica a ser incorporada na avaliação da QVRS de pacientes com DRC.

Diante do exposto, percebe-se que o número de estudos abordando a ativação de pessoas com DRC é reduzido, e nem todos os estudos avaliaram as mesmas variáveis, o que impossibilita a generalização dos dados referentes a essa temática. Além disso, as inconsistências encontradas entre alguns fatores associados a maiores níveis ou escores de ativação podem desencorajar os profissionais de saúde a abordarem determinadas variáveis no planejamento de intervenções que visam melhorar os níveis de ativação do paciente com DRC.

Contudo, conhecer o nível de ativação do paciente com DRC e seus fatores associados traz implicações para a prática clínica dos profissionais de saúde, uma vez que, de posse desse conhecimento, pode-se traçar um plano de cuidados individualizado, colaborativo e que favoreça o autogerenciamento da doença e de seu tratamento.

Salienta-se que, ao trabalhar com o conceito de ativação do paciente, o profissional de saúde considerará os conhecimentos, as habilidades, a motivação e as crenças do indivíduo para o engajamento do autocuidado ${ }^{11}$. Assim promoverá a educação em saúde através das particularidades do indivíduo no que diz respeito ao seu engajamento com a saúde e à capacidade para a autogestão.

Para concluir, na maior parte dos estudos, os pacientes renais crônicos foram classificados como alta ativação (níveis 3 e 4). A ativação do paciente renal crônico foi associada a múltiplos fatores, sendo eles idade, escolaridade, condição socioeconômica, realização de atividades de lazer e do autocuidado, índice de massa corporal, presença de sintomas, depressão, percepção da doença, saúde física, suporte social, suporte clínico e qualidade de vida relacionada à saúde.

Salienta-se que conhecer os fatores associados ao nível de ativação do paciente com DRC propicia o direcionamento da atuação profissional junto ao paciente, visando aumentar e/ou manter o paciente altamente ativado para que possa, assim, desfrutar dos benefícios da alta ativação.

Recepción: 01-10-20

Aceptación: 15-01-21

Publicación: 30-03-21

\section{Bibliografía}

1. Barreto Marinho AWG, da Paz Penha A, Tolentino Silva M, Freire Galvão T. Prevalência de doença renal crônica em adultos no Brasil: revisão sistemática da literatura. Cad. Saúde colet. 2017 Jul-Set;25(3):379-88.

2. Saldanha Thomé $F$, Cintra Sesso $R$, Lopes $A A$, Lugon JR, Tzanno Martins $C$. Inquérito Brasileiro de Diálise Crônica 2017. J Bras Nefrol. 2019 AbrJun;41(2):208-14.

3. Alcalde PR, Mastroianni Kirsztajn G. Gastos do Sistema Público de Saúde com doença renal crônica. J. Bras. Nefrol. 2018 Abr-Jun;40(2):1-8.

4. Berger Oliveira AP, Berger Schmidt D, Malucelli Amatneeks T, dos Santos JC, Raizz Cavallet LH, Michel RB. Qualidade de vida em pacientes em hemodiálise e a relação com mortalidade, hospitalizações e baixa adesão ao tratamento. J. Bras. Nefrol. 2016 Out-Dez;38(4):411-20.

5. Filgueiras de Assis Mello MV, Angelo M. Impacto da doença renal crônica: experiências de pacientes e familiares do extremo Norte do Brasil. Invest. 
Educ. Enferm. 2018 [consultado 29 jul 2020]; 36(1): [aprox. 10 p.]. Disponível em: http://dx.doi. org/10.17533/udea.iee.v36nle02.

6. Insignia Health. Increasing Activation Starts with Measurement. Insignia Health. [consultado 30 dez 2020]. Disponível em: https://s3.amazonaws.com/insigniahealth. com-assets/PAM-Fact-Sheet. 20200505. pdf? $\mathrm{mtime}=20200505094829 \&$ focal $=$ none .

7. Sacks RM, Greene J, Hibbard J, Overton V, Parrotta CD. Does patient activation predict the course of type 2 diabetes? A longitudinal study. Patient Educ Couns. $2017 \mathrm{Jul} ; 100(7): 1268-75$.

8. Sacks RM, Greene J, Hibbard J, Overton V. How well do patient activation scores predict depression outcomes one year later? J Affect Disord. 2014 Dez;169:1-6.

9. Bos-Touwen I, Schuurmans M, Monninkhof $E_{\text {, }}$ Korpershoek Y, Spruit-Bentvelzen L, Ertugrul I, et al. Patient and Disease Characteristics Associated with Activation for Self-Management in Patients with Diabetes, Chronic Obstructive Pulmonary Disease, Chronic Heart Failure and Chronic Renal Disease: A Cross-Sectional Survey Study. PloS one. 2015 [consultado 20 mai 2020]; 10(5): [aprox. 16 p.]. Disponível em: https://doi.org/10.1371/journal. pone.0126400.

10. Bodenheimer T, Lorig K, Holman H, Grumbach K. Patient Self-management of Chronic Disease in Primary Care. JAMA. 2002 Nov;288(19):246975.

11. Hibbard JH, Stockard J, Mahoney ER, Tusler M. Development of the Patient Activation Measure (PAM): conceptualizing and measuring activation in patients and consumers. Health Serv Res. 2004 Jun;39(4):1005-26.

12. Hibbard J, Greene J. What the evidence shows about patient activation: better health outcomes and care experiences; mewer data on costs. Health affairs. 2013 [consultado 12 mai 2020]; 32: [aprox. 8 p.]. Disponível em: https://doi.org/10.1377/ hlthaff.2012.1061.

13. Insignia Healht. Patient Activation Measure ${ }^{\circledR}$ $\left(\right.$ PAM $^{\circledR}{ }^{\circledR}$. [consultado 15 mar 2020]. Disponível em: https://s3.amazonaws.com/insigniahealth. com-assets/PA M-Fact-Sheet.20200505. pdf? $\mathrm{mtime}=20200505094829 \&$ focal $=$ none .

14. Hibbard JH, Mahoney ER, Stockard J, Tusler M. Development and testing of a short form of the patient activation measure. Health Serv Res. 2005 Fev; 40(6 Pt 1):207-14.

15. Greene J, Hibbard JH, Sacks R, Overton V, Parrotta $C D$. When patient activation levels change, health outcomes and costs change, too. Health Affairs. 2015 [Consultado 08 ago 2020]; 34: [aprox. 8 p.]. Disponível em: https://doi.org/10.1377/ hlthaff.2014.0452.

16. Hibbard JH, Mahoney E, Sonet E. Does patient activation level affect the cancer patient journey? Patient Educ Couns. 2017 100(7):1276-79.

17. Zimbudzi E, Lo C, Ranasinha S, Fulcher GR, Jan S, Kerr PG, et al. Factors associated with patient activation in an Australian population with comorbid diabetes and chronic kidney disease: a cross sectional study. BMJ Open. 2017 [Consultado 05 mai 2020]; 7(10): [aprox. 9 p.]. Disponível em: https://doi.org/10.1136/bmjopen-2017-017695.

18. Greene J, Hibbard JH. Why does patient activation matter? An examination of the relationships between patient activation and health-related outcomes. J Gen Intern Med. 2012 Nov;27(5):520-6.

19. Souza MT, Silva MD, Carvalho R. Integrative review: what is it? How to do it?. Einsten. 2010 [consultado 29 mai 2020]; 8(1): [aprox. 5 p.]. Disponível em: https://doi.org/10.1590/s1679-45082010rw1134.

20. Principais itens para relatar Revisões sistemáticas e Meta-análises:Arecomendação PRISMA.Epidemiol. Serv. Saúde. 2015 [consultado 29 jan 2020]; 24(2): [aprox. 8 p.]. Disponível em: http://www.scielo. $\mathrm{br} / \mathrm{scielo}$.php? script=sci_arttext\&pid=S223796222015000200335\&lng=en. http://dx.doi. org/10.5123/S1679-49742015000200017.

21. Velez-Bermúdez M, Christensen AJ, Kinner EM, Roche AL, Fraer M. Exploring the Relationship Between Patient Activation, Treatment Satisfaction, and Decisional Conflict in Patients Approaching End-Stage Renal Disease. Ann Behav Med. 2018 Set;53(9):816-26. 
22. Nelson R, Pankratz V, Ghahate $D$, Bobelu J, Faber $\mathrm{T}$, Shah V. Home-based kidney care, patient activation, and risk factors for CKD progression in Zuni Indians: a randomized, controlled clinical trial. Clin J Am Soc Nephrol. 2018 [consultado 15 ago 2020]; (12): [aprox. 9 p.]. Disponível em: https:// doi.org/10.2215/CJN.06910618.

23. Johnson $M L$, Zimmerman $L$, Welch $\mathrm{JL}$, Hertzog M, Pozehl B, Plumb T. Patient activation with knowledge, self-management and confidence in chronic kidney disease. J Ren Care. 2016 Mar; 42(1):15-22.

24. Lo C, Zimbudzi E, Teede HJ, Kerr PG, Ranasinha $S$, Cass $A$, et al. Patient-reported barriers and outcomes associated with poor glycaemic and blood pressure control in co-morbid diabetes and chronic kidney disease. J Diabetes Complications. 2019 Jan; 33(1):63-8.

25. Zimbudzi E, Lo C, Ranasinha S, Kerr PG, Polkinghorne $K R$, Teede $H$, et al. The association between patient activation and self-care practices: A cross-sectional study of an Australian population with comorbid diabetes and chronic kidney disease. Health Expect. 2017 [consultado 01 mar 2020]; 20(6): [aprox. 10 p.]. 1375-84. Disponível em: https://doi.org/10.1111/hex.12577.

26. Van Bulck L, Claes K, Dierickx K, Hellemans A, Jamar S, Smets, et al. Patient and treatment characteristics associated with patient activation in patients undergoing hemodialysis: a cross-sectional study. BMC Nephrol. 2018 [consultado 15 mai 2020]; (1): [aprox. 9 p.]. Disponível em: https://doi. org/10.1186/s12882-018-0917-2.

27. The Renal Association. Transforming participation in chronic kidney disease. Bristol; 2019 [consultado 06 jun 2020]; Disponível em: https://www.thinkkidneys. nhs.uk/ckd/wp-content/uploads/sites/4/2019/01/ Transforming-Participation-in-Chronic Kidney-Dis.

28. NQF. National Quality Forum: Quality positioning system. 2016 [consultado 02 jan 2021]. Disponível em: file://C:/Users/user/Downloads/pfcc_20152017_final_report.pdf.

29. Cameron J, Worrall-Carter L, Riegel B, Lo SK, Stewart S. Testing a Model of Patient Characteristics, Psychologic Status, and Cognitive
Function as Predictors of Self-Care in Persons with Chronic Heart Failure. Heart lung. 2009 Fev; 38(5):410-8.

30. Tusa N, Kautiainen $H$, Elfving $P$, Sinikallio $S$, Mäntyselkä P. Relationship between patient activation measurement and self-rated health in patients with chronic diseases. BMC Fam Pract. 2020 [consultado 09 jan 2021]; 21(1): [aprox. 8 p.]. Disponível em: https://doi.org/10.1186/s12875020-01301-y.

31. Dunlay SM, Griffin JM, Redfield MM, Roger VL. Patient Activation in Acute Decompensated Heart Failure. J Cardiovasc Nurs. 2017 [consultado 11 jan 2021]; 32(6): [aprox. 8 p.]. Disponível em: https:// doi.org/10.1097/JCN.000000000000036.

32. Shortell S, Poon BY, Ramsay $P$, Rodriguez $H$, Ivey $S$, Huber $T$, et al. A multilevel analysis of patient engagement and patient-reported outcomes in primary care practices of accountable care organizations. J Gen Intern Med.2017 [consultado 18 set 2020]; (6): [aprox. 8 p.]. Disponível em: https://doi.org /10.1007/s11606-016-3980-z.

33. McCabe PJ, Stuart-Mullen LG, McLeod CJ, 0 Byrne T, Schmidt MM, Branda ME, et al. Patient activation for self-management is associated with health status in patients with atrial fibrillation. Patient Prefer Adherence. 2018 [consultado 25 set 2020]; 21(225): [aprox. 10 p.]. Disponível em: https://doi.org/10.2147/PPA.S172970.

34. Yadav UN, Lloyd J, Hosseinzadeh $\mathrm{H}$, Baral KP, Bhatta N, Harris MF. Levels and determinants of health literacy and patient activation among multimorbid COPD people in rural Nepal: Findings from a cross-sectional study. Plos One. 2020 [consultado 30 dez 2020]. 15(5): [aprox. 16 p.]. Disponível em: https://doi.org/10.1371/journal.pone.0233488.

35. Zeng $H$, Jiang $R$, Zhou M., Wu L, Tian B, Zhang $Y$ et al. Measuring patient activation in Chinese patients with hypertension and/or diabetes: reliability and validity of the PAM13. J Inter Med Resea. 2019 [consultado 26 set 2020]; 47(12): [aprox. 10 p.]. Disponível em: https://doi. org/10.1177/0300060519868327.

36. Rask KJ, Ziemer DC, Kohler SA, Hawley JN, Arinde FJ, Barnes CS. Patient Activation Is Associated 
With Healthy Behaviors and Ease in Managing Diabetes in an Indigent Population. Diabetes Educ. 2009 Jul-Aug;35(4):622-30.

37. Gleason KT, Tanner EK, Boyd CM, Saczynski JS, Szanton SL. Factors associated with patient activation in an older adult population with functional difficulties. Paciente Educ Couns. 2016 Ago; 99(8):1421-6.

38. Lubetkin EI, Lu WH, Gold MR. Levels and correlates of patient activation in health center settings: building strategies for improving health outcomes. J Health Care Poor Underserved. 2010 Ago;21(3):796-808.

39. Toobert DJ, Hampson SE, Glasgow RE. The summary of diabetes self-care activities measure: results from 7 studies and a revised scale. Diabetes Care. 2000 [consultado 09 jan 2021]; 23(7): [aprox. 8 p.]. Disponível em: https://doi.org/10.2337/ diacare.23.7.943.

40. Paliative Care Outcome Scale. POS S - Renal. [consultado 6 jun 2020]; Disponível em: https://pospal. org/mdlx/login/confirm.php?data=el RmqlkoORLjFvS/de_rocha\%40ymail\%2Ecom.

41. Golubinski V, Oppel EM, Schreyög J. A systematic scoping review of psychosocial and psychological factors associated with patient activation. Patient Educ Couns. 2020 Out;103(10):2061-8.

42. Korpershoek Y, Bos-Touwen ID, de Man-van Ginkel JM, Lammers JW, Schuurmans MJ, Trappenburg J. Determinants of activation for self-management in patients with COPD. Int $\mathrm{J}$ Chron Obstruct Pulmon Dis. 2016 [consultado 9 ago 2020]; 1(11): [aprox. 10 p.]. 1757-66. Disponível em: https://doi. org/10.2147/COPD.S109016.
43. Van den Bosch J, Warren DS, Rutherford PA. Review of predialysis education programs: a need for standardization. Patient Prefer Adherence. 2015 [consultado 7 out 2020]; 2015(9): [aprox. 13 p.]. Disponível em: https://doi.org/10.2147/ppa. s81284.

44. Hall G, Bogan A, Dreis S, Duffy A, Greene S, Kelley $K$, et al. New directions in peritoneal dialysis patient training. Nephrol Nurs J. 2004 Mar-Abr; 31(2):159-63.

45. Herdman M, Gudex C, Lloyd A, Janssen MF, Kind $P$, Parkin $D$, et al. Development and preliminary testing of the new five-level version of EQ-5D (EQ5D-5L). Qual Life Res. 2011; [consultado 27 set 2020]; 20(10): aprox. 10 p.]. 1727-36. Disponível em: https://doi.org/10.1007/s11136-011-9903-x.

46. Brooks R. EuroQol: the current state of play. Health Policy. 1996 [consultado 27 set 2020]; 37(1): [aprox. 10 p.]. Disponível em: https://doi. org/10.1016/0168-8510(96)00822-6.

47. Measuring Dialysis Patients' Health-Related Quality of Life with the KDQOL-36. [consultado 30 jul 2020]. Disponível em: https://www.kdqol-complete. org/pdfs/kdqol-36.pdf.

48. Blakemore $A$, Hann $M$, Howells $K$, Panagioti $M$, Sidaway $M$, Reeves $D$ et al. Patient activation in older people with long-term conditions and multimorbidity: correlates and change in a cohort study in the United Kingdom. BMC Health Serv Res. [Internet]. 2016; [consultado 21 set 2020]; 18;16 (1): [aprox. 11 p.]. DOI: https://doi.org/10.1186/ s12913-016-1843-2.

Este artículo se distribuye bajo una Licencia Creative Commons Atribución-NoComercial 4.0 Internacional. https://creativecommons.org/licenses/by-nc/4.0/

Open Access (C) (1) (8) 